Taipe-Nasimba, N.; Cantón Chirivella, E. (2020) Street Workout: Psychosocial Profile of Its Practitioners. Revista Internacional de Medicina y Ciencias de la Actividad Física y el Deporte vol. 20 (79) pp. 521-534 Http://cdeporte.rediris.es/revista/revista79/artstreet1122.htm

DOI: http://doi.org/10.15366/rimcafd2020.79.009

\title{
ORIGINAL
}

\section{STREET WORKOUT: PERFIL PSICOSOCIAL DE SUS PRACTICANTES}

\section{STREET WORKOUT: PSYCHOSOCIAL PROFILE OF ITS PRACTITIONERS}

\author{
Taipe-Nasimba, N. ${ }^{1}$ y Cantón Chirivella, E. ${ }^{2}$ \\ ${ }^{1}$ Doctora. Facultad de Psicología. Unidad de Investigación "Salud, motivación y deporte". \\ Universidad de Valencia (España) notaina@alumni.uv.es \\ ${ }^{2}$ Doctor. Facultad de Psicología. Universidad de Valencia (España) enrique.canton@uv.es
}

Código UNESCO / UNESCO Code: 6114.17 Percepciones y movimientos sociales / Social perceptions and movements

Clasificación Consejo de Europa / Council of Europe Classification: 16. Sociología del deporte / Sociology of sport

Recibido 10 de abril de 2018 Received April 10, 2018

Aceptado 24 de mayo de 2020 Accepted May 24, 2020

\section{RESUMEN}

La presente investigación estudia las principales características psicológicas de los practicantes de Street Workout, una modalidad deportiva emergente y en auge en los últimos años. A través de un estudio descriptivo-correlacional, con una muestra de 107 practicantes de España, se elaboró un estudio piloto del perfil psicosocial que incluye la evaluación de los motivos de práctica, la satisfacción muscular, la cohesión y el bienestar psicológico; además de una comparación en función de si compiten. Los resultados señalan que es un deporte practicado mayoritariamente por hombres menores de 23 años, cuya principal motivación es hallar un equilibrio entre la preocupación por la salud y su aspecto físico. Los niveles en cohesión y bienestar psicológico son altos, y bajos en cuatro de las cinco dimensiones de la satisfacción muscular. Por consiguiente, los datos de esta primera aproximación apoyan una visión positiva de las características psicológicas de los practicantes de Street Workout de la muestra.

PALABRAS CLAVE: Street Workout, motivación, bienestar, cohesión, satisfacción muscular. 


\section{ABSTRACT}

This research studies the main psychological characteristics of Street Workout practitioners, an emerging and booming sports modality in recent years. Through a descriptive and correlational study, with a sample of 116 practitioners from Spain, a psycho social profile was developed and includes the evaluation of the reasons for the practice, muscle appearance satisfaction, the cohesion and the psychological well-being; in addition, includes a comparison based on whether they compete. The results suggest that it is a male-dominated sport, all under 23 years old, whose main motivation is to find a balance between the concern about the health and their physical appearance. The levels of cohesion and psychological well-being are high, and low in four of the five dimensions of muscle satisfaction. Therefore, the data from this first approach support a positive view of the psychological characteristics of Street Workout practitioners and its practice.

KEY WORDS: Street Workout, motivation, well-being, cohesion, muscle satisfaction

\section{INTRODUCCIÓN}

En los últimos años está teniendo lugar el crecimiento y expansión de nuevas modalidades de práctica deportiva que están transformando y generando nuevos grupos de aficionados y deportistas (Maza et al., 2011). Dentro de éstas, destaca el Street Workout, como un fenómeno de rápida expansión a nivel internacional, consiguiendo millones de seguidores activos alrededor de todo el mundo en pocos años, además de lograr una creciente popularidad entre los más jóvenes (World Street Workout and Calisthenics Federation, WSWCF, 2018).

El Street Workout es una modalidad de entrenamiento al aire libre que utiliza el equipamiento e infraestructuras urbanas (disponibles en algunos parques 0 playas, por ejemplo) como las barras, dominadas de diferentes alturas, paralelas o zonas de anillas. Se basa en los principios de entrenamiento de la calistenia, que busca la mejora de la forma física y la salud a partir de la realización de ejercicios orientados a trabajar la aptitud musculo-esquelética, que engloba dos cualidades físicas básicas: la fuerza muscular (resistencia, potencia e hipertrofia) y el equilibrio (estabilidad escapular, lumbopélvica y en apoyo de manos) (Asociación Cultural y Recreativa Street Workout Zaragoza, 2015; Monje, 2017; Pérez, 2016).

Las rutinas de SW suelen ser dinámicas y es frecuente que incluyan elementos acrobáticos o ejercicios propios de la gimnasia deportiva, además de ejercicios que contribuyen al fortalecimiento de la salud, al desarrollo de la fuerza, de la resistencia, y de la flexibilidad (Pérez, 2016). Mediante los ejercicios que se realizan en el SW se busca el desarrollo de las habilidades motrices de los practicantes, en las que además es muy importante conocer el propio cuerpo y trabajar su control, es decir el entrenamiento cognitivo. El trabajo realizado a través de un entrenamiento continuado de SW y su combinación con el 
entrenamiento cognitivo, permitirán la posterior realización de ejercicios en los que la intensidad, entendida como el grado de esfuerzo exigido, sea cada vez mayor (Federación Española de Street Workout y Calistenia, FESWC, 2017; Jiménez, 2006).

Su práctica, además del entrenamiento en flexibilidad, fuerza y equilibrio, implica una filosofía y un estilo de vida cuyas bases se fundamentan en la práctica de ejercicio físico regular y el seguimiento de hábitos de vida saludables complementarios. Asimismo, transmite los valores propios de cualquier deporte de una forma explícita y consciente, como el respeto, la persistencia, la constancia, el esfuerzo, la ayuda mutua, el compañerismo y la superación personal (WSWCF, 2017). A pesar de tratarse de un deporte de realización individual, el grupo adquiere una notoria importancia como fuente de transmisión de conocimiento, de apoyo y de instrucción, por lo que la cohesión es uno de sus factores clave como proceso dinámico, que se refleja en la tendencia del grupo a mantenerse unidos en la consecución de objetivos instrumentales y/o para la satisfacción de las necesidades afectivas de los miembros (WSWCF, 2017; RTVE, 2014).

El origen de este fenómeno es difuso, sin embargo, se asocia a la cultura hiphop que se basa en salir a la calle y ejercitarse al aire libre con cualquier cosa que se pudiera encontrar, llevando el concepto de "gimnasio" a cualquier parte de la ciudad (Rodríguez, 2014; RTVE, 2014). Una de las primeras referencias fiables sobre su origen se remonta al año 2002 en Estados Unidos, en un DVD titulado "Thug Workout: Fitness From the Streets". La expansión de esta modalidad deportiva fue impulsada en gran medida por la aparición de YouTube en 2005, alcanzando una difusión a nivel mundial, sobre todo en América del Norte y en Europa Occidental (Pérez, 2016), a través de las plataformas digitales y las redes sociales.

En España, el primer grupo de entrenamiento surge en el año 2010 y se denomina Barbarrio, que se autodefine como "un colectivo socio-deportivo que se reúne para entrenar ejercicio físico en la calle, desarrollando esta actividad bajo los siguientes principios: vida sana, solidaridad, diversión e integración" (Pérez, 2016, p. 2). Adoptan un discurso congruente con los valores positivos asociados a la práctica deportiva y su función como parte fundamental en la formación integral del ser humano (Gutiérrez Sanmartín, 2004) y contribuye a generar herramientas que ayudan a las personas a desenvolverse en la vida (UNICEF, 2011).

Los efectos positivos de la práctica deportiva general a nivel físico, psicológico y social, han sido ampliamente descritos hasta la fecha, considerándose como un elemento fundamental para todos los colectivos de la sociedad (Huebner, 2004; Heinemann, 2002: Pulgar y Fernández-Luna, 2019). Sin embargo, tal y como afirman Caracuel y Arbinaga (2010) o Arbinaga y Cantón (2013), al igual que ocurriría en cualquier otro deporte, los efectos beneficiosos de su práctica no se derivan de la mera práctica de ejercicio, sino que estos proceden en gran medida de la adopción de hábitos de vida que se promueven conscientemente a través de dicha práctica y del tipo de deporte que se realice. En la dimensión psicológica se ha hallado una correlación entre la práctica deportiva regular y los índices de 
salud mental más relevantes en la actualidad: el bienestar psicológico y la mejora del auto-concepto (Blacklock, Rhodes y Brown, 2007).

Por otra parte, el contacto social a través del deporte es un arma de doble filo, también puede contener un polo negativo (Djalal, 2015). Del mismo modo que posee el potencial para promover la integración y cooperación, a través del tipo de interacción que se dé en la práctica, ésta puede contribuir a la creación de conflictos, a la exclusión, la discriminación (Elling y Claringbould, 2005) o a la extensión de estereotipos acerca de los grupos minoritarios (Walseth y Fasting, 2004). El entorno en el que se realiza la práctica deportiva influye en el tipo de interacción que tiene lugar, llegando a fomentar conductas violentas o los comportamientos que van en contra de la integración, incluso puede relacionarse con conductas de preservación de la identidad étnica de un determinado grupo frente a otros, dando como resultado situaciones de enfrentamiento 0 aislamiento (Stodolska y Alexandris, 2004); también podría potenciar comportamientos relacionados con el abuso de alcohol o drogas (Acero et al., 2002; Wang et al., 2014).

Además, ha de tenerse en cuenta que en la sociedad actual el aspecto físico se identifica cada vez más con el éxito, el autocontrol y otras cualidades positivas (Rodríguez, 2007); lo que puede llevar a un culto obsesivo por el ideal promovido por el entorno. En algunas ocasiones surgen fácilmente sentimientos de insatisfacción corporal y personal, que pueden reflejarse en ansiedad social y una necesidad imperiosa de cambio físico (Rodríguez y Esnaola, 2009) a través del ejercicio físico compulsivo o el uso de sustancias que faciliten la definición y el aumento de la musculatura con rapidez. En los últimos años, las investigaciones se han centrado en los ejercicios relacionados con el entrenamiento de contra-resistencia o entrenamiento muscular (Cantón y Arbinaga, 2013), por su propensión a desarrollar este tipo de conductas (Ramos, Sansebastián y Madoz, 2001), que son mayores en los competidores (Arbinaga y Caracuel, 2007).

Queda clara, por lo tanto, la dualidad del deporte y la necesidad la promoción de una práctica que se lleve a cabo desde una perspectiva que promueva sus beneficios; profundizando en el conocimiento de las nuevas modalidades deportivas orientadas a valores positivos y deseables socialmente.

Como respuesta a la falta de literatura científica respecto a temas relacionados con nuevas modalidades deportivas en auge, en el presente trabajo se pretende contribuir al conocimiento de esta nueva modalidad a través de la elaboración del estudio piloto del perfil psicosocial de sus practicantes en la zona de Valencia; valorando diferentes variables psicológicas: motivos de práctica, cohesión, bienestar psicológico y satisfacción muscular. Además, se comparan las diferencias entre grupos, según si participan o no en competiciones y en base a su situación migratoria. 


\section{MATERIAL Y MÉTODOS}

Se ha empleado una metodología cuantitativa que corresponde a estudio no experimental de carácter transversal, descriptivo-correlacional, que nos ha permitido elaborar el estudio piloto del perfil psicosocial de los sujetos de esta muestra.

\section{PARTICIPANTES}

La muestra estuvo compuesta por un total de 107 practicantes de Street Workout, con edades comprendidas entre los 18 y 34 años $(M=20,7 ; D T=4)$, residentes en España. El muestreo llevado a cabo fue el no probabilístico accidental de población a disposición, con un criterio único de inclusión: practicar de manera regular Street Workout (entre dos y tres veces a la semana, un mínimo de una hora cada sesión).

\section{INSTRUMENTOS}

La selección de instrumentos corresponde a la identificación de los mejores medios de evaluación disponibles en nuestro idioma y entorno cultural, una vez establecidas las variables de estudio, partiendo de los trabajos y estudios previos (Arbinaga y Cantón, 2013; Taipe, Peris y Cantón, 2019).

Cohesión. Para medir esta variable se empleó la versión española del Multidimensional Sport Cohesion Instrument (MSCl; Andrade, Arce y Seoane, 2002). Constituido por 22 ítems dispuestos en las cuatro dimensiones: calidad de trabajo respecto a la ejecución, roles valorados, unidad de propósito y atracción hacia el grupo. Las opciones de respuesta van desde 0 a 10. Los coeficientes de fiabilidad oscilan entre 0,92 y 0,75 .

Satisfacción muscular. Se empleó la adaptación española del Muscle Appearance Satisfaction Scale (MASS; González-Martí et al., 2011). Está formada por 19 ítems dicotómicos repartidos en cinco factores: dependencia del culturismo, conductas de verificación muscular, uso de sustancias, lesión y satisfacción muscular. El coeficiente de fiabilidad es 0,71 .

Bienestar psicológico. Se utilizó la adaptación española de la Escala de Bienestar Psicológico de Ryff (Díaz et al,, 2006), formada por 29 ítems distribuidos en seis factores: auto-aceptación, relaciones positivas, autonomía, dominio del entorno, propósito en la vida y crecimiento personal. Las opciones de respuesta van desde 1 a 6 . El alfa de Cronbach oscilan entre 0,81 y 0,72, salvo en Autonomía y Dominio del entorno, con 0,54 y 0,62 respectivamente.

Datos sociodemográficos. Se completó la información con datos sobre su edad, sexo, país de nacimiento y residencia, así como su situación laboral. Se incluyeron también datos procedentes de la actividad deportiva, así como de los principales motivos de inicio y permanencia en ella. 


\section{PROCEDIMIENTO}

Se acudió a los lugares de práctica habitual de Street Workout, los parques de entrenamiento localizados en las zonas del centro de la ciudad de Valencia, como puntos más frecuentados. Informados los participantes de la finalidad del estudio, se obtuvo su consentimiento libre e informado, garantizando además el anonimato de su colaboración. Los participantes contestaron todos los ítems en presencia de los investigadores. El tiempo aproximado que necesitaron fue de 20 minutos. Adicionalmente, se creó una versión digital del instrumento en la plataforma Google Docs Form, cuyo acceso se realiza a través de un enlace privado enviado por correo electrónico y al que tenían acceso exclusivamente las personas interesadas en el estudio y referidas por los mismos participantes. Facilitamos este instrumento a través de redes sociales y grupos dedicados a la práctica de SW en toda España. En este caso, se manifestó la disponibilidad para aclarar cualquier tipo de dudas o consultas que les surgieran a través del correo electrónico. El $65 \%$ de los participantes contestó a través de la plataforma digital, mientras que el $35 \%$ restantes lo hizo de manera tradicional, no hallándose diferencias en la forma de respuesta.

\section{ANÁLISIS ESTADÍSTICOS}

Para establecer el perfil psicosocial se realizaron de entrada análisis descriptivos (frecuencia, medias y desviaciones típicas) de las variables sociodemográficas, los datos relacionados con la actividad física, los motivos de inicio y permanencia en la práctica y en las variables psicológicas antes mencionadas: cohesión, bienestar psicológico y satisfacción muscular. Se realizaron también comparaciones de medias para conocer las diferencias entre grupos, en función de si competían o no y de si constituían parte de la población inmigrante o no lo eran. Se aplicó la prueba no paramétrica $U$ de Mann-Whitney para muestras independientes en la comparación de variables cuantitativas, analizado mediante el programa estadístico SPSS versión 20.0.

\section{RESULTADOS}

Los resultados obtenidos revelan que el $96,3 \%$ de los participantes son hombres, con edades comprendidas entre los 19 y los 23 años (49,5\%), que llevan practicando esta modalidad deportiva una media de 9,4 meses $(D T=7,11)$. La mayoría de los participantes son estudiantes $(68,2 \%)$, el $16{ }^{\prime} 8 \%$ trabaja, el $5,6 \%$ estudia y trabaja, y un $9,3 \%$ que se halla en situación de desempleo. Se encontró que el $21 \%$ de la muestra son inmigrantes.

Respecto a la información relacionada con el Street Workout, los datos señalan que lo conocieron predominantemente a través de amigos/conocidos (46\%), de redes sociales como Facebook o YouTube (44\%) y por la observación de otras personas en parques cercanos (10\%). Los datos indican que la gran mayoría $(68,2 \%)$ afirma practicar adicionalmente otros deportes complementarios al SW, como fútbol, atletismo, ciclismo o artes marciales, entre los más señalados. 
Los datos sobre los motivos considerados como importantes para el inicio en su práctica del Street Workout más frecuentes entre los participantes son: mejorar la condición física $(88,4 \%)$, diversión $(80,6 \%)$, aumentar la musculatura $(73,9 \%)$ y mejorar la imagen corporal $(70,7 \%)$. En cuanto a los motivos de permanencia, encontramos que son: la mejora de la condición física $(82,2 \%)$ y la diversión $(67,3 \%)$ como los más frecuentes.

Los resultados obtenidos sobre la cohesión (Tabla 1) muestran altas puntuaciones en sus cuatro dimensiones, lo que refleja la tendencia del grupo a mantenerse unidos. Respecto a la satisfacción muscular, las puntuaciones son bajas en Verificación Muscular ( $X=0,22 ; D T=0,27)$, Uso de Sustancias $(X=0,27$; $D T=0,22)$ y Dependencia $(X=0,3 ; D T=0,23)$; lo que indica una baja frecuencia de conductas de verificación del tamaño de los músculos, un uso y/o admisión de suplementos para lograr un mayor crecimiento de los músculos no generalizados en esta muestra, y una baja necesidad de practicar ejercicio de manera compulsiva, respectivamente. Por el contrario, hallamos puntuaciones moderadas/bajas en Lesión ( $X=0,41 ; D T=0,35)$, lo que indica que para parte de nuestra muestra el dolor forma parte del entrenamiento, aunque no es una postura generalizada. Finalmente, las puntuaciones en Satisfacción Muscular $(X=0,7 ; D T=0,36)$ son altas, reflejando que los participantes me muestran satisfechos con el tamaño y forma de su musculatura. Todo ello refleja una satisfacción con la musculatura extendida, sin la presencia de datos que señalen la presencia de algún tipo de problema o trastorno relacionado con ello.

Sobre las medias obtenidas en el Bienestar Psicológico, los datos señalan que los practicantes muestran puntuaciones moderadas/altas en las seis dimensiones del instrumento (Tabla 1). Lo que indica que los participantes de la presente investigación presentan niveles altos en esta variable.

Tabla 1. Descriptivos de los factores de la escala MSCl y Bienestar Psicológico

\begin{tabular}{ccc}
\hline & Media & DT \\
\hline Unidad de propósitos & 8,5 & 1,87 \\
Roles valorados & 8,5 & 2,19 \\
Atracción al grupo & 8,8 & 1,18 \\
Calidad de trabajo & 8,1 & 2,1 \\
\hline & Media & DT \\
\hline Aceptación & 5,0 & 0,89 \\
Autonomía & 4,5 & 0,82 \\
Relaciones positivas & 4,8 & 1,05 \\
Dominio del entorno & 4,0 & 0,75 \\
Propósito de vida & 4,9 & 0,82 \\
Crecimiento personal & 5,1 & 0,81 \\
\hline
\end{tabular}

Respecto a las comparaciones entre grupos, la prueba $U$ de Mann-Whitney mostró diferencias estadísticamente significativas entre los competidores y los no-competidores, en las dimensiones que se muestran en la Tabla 2. Aquellos participantes que no compiten son los que presentan una mayor puntuación en todas las dimensiones, con diferencias significativas. 
Tabla 2. Diferencias significativas entre Competidores y No Competidores.

\begin{tabular}{ccccccc}
\cline { 2 - 6 } & \multicolumn{2}{c}{$\begin{array}{c}\text { No Competidores } \\
(\mathrm{n}=35)\end{array}$} & \multicolumn{2}{c}{$\begin{array}{c}\text { Competidores } \\
(\mathrm{n}=72)\end{array}$} & & \\
\cline { 2 - 6 } & Media & $\mathbf{D T}$ & Media & DT & $\mathbf{Z}$ & $\mathbf{p}$ \\
\hline Aceptación (Bienestar) & 4,9 & 0,90 & 5,2 & 0,85 & $-2,12$ & 0,033 \\
$\begin{array}{c}\text { Autonomía (Bienestar) } \\
\text { Dominio del Entorno } \\
\text { (Bienestar) }\end{array}$ & 4,4 & 0,80 & 4,8 & 0,77 & $-2,79$ & 0,005 \\
$\begin{array}{c}\text { Propósito de } \\
\text { Vida(Bienestar) }\end{array}$ & 3,8 & 0,72 & 4,3 & 0,69 & $-2,93$ & 0,003 \\
$\begin{array}{c}\text { Unidad de Propósitos } \\
\text { (MSCl) }\end{array}$ & 8,8 & 0,80 & 5,1 & 0,82 & $-2,16$ & 0,030 \\
\hline
\end{tabular}

Nota: Nivel de significación $p<0,05$

\section{DISCUSIÓN}

Esta investigación planteó como objetivo el estudio piloto del perfil psicosocial de los practicantes de Street Workout en la ciudad de Valencia. La pretensión ahondar en el conocimiento de los practicantes de este deporte en la muestra recogida, tomando como variables principales la cohesión, bienestar psicológico y satisfacción muscular. Estos constructos se eligieron teniendo en cuenta las características del Street Workout. Por lo que, al tratarse de un deporte individual con un importante componente grupal, se consideró oportuno evaluar el nivel de cohesión en los grupos de entrenamiento. Además, al tratarse de una modalidad con una parte orientada al desarrollo de fuerza muscular, se valoró la relevancia de incluir la evaluación de la satisfacción muscular en los participantes y descartar la presencia de indicios de dependencia a la actividad. Finalmente, se incluyó el bienestar psicológico como índice de salud mental relacionado con la práctica deportiva.

Los datos del perfil sociodemográfico ponen de manifiesto que los participantes de esta investigación son mayoritariamente hombres jóvenes. Esto refuerza lo que otros estudios han encontrado sobre la práctica deportiva según el género, siendo las mujeres en la que se halla un menor implicación en actividades físicodeportivas, principalmente en disciplinas relacionadas con el entrenamiento en musculación (García, 2006) o actividades músculo-esqueléticas (Zaragoza, Serrano y Generelo, 2004). Como ocurre con otras actividades físico-deportivas como el Fisicoculturismo, una posible explicación es que el SW al trabajar la fuerza sea visto como una actividad masculina que amenaza ese ideal del cuerpo femenino y feminidad, por lo que las mujeres opten por realizar otro tipo de actividades, si bien puede ser un patrón cultural que se vaya modificando con el tiempo (Sicilia et al., 2009).

El $77,6 \%$ de los participantes no forma parte del mercado laboral (estudiantes y desempleados) y un $21 \%$ son inmigrantes, constituyéndose parte de sectores de la población más vulnerables al sedentarismo (Taverno et al., 2014) y, por ende, grupos con mayor riesgo a sufrir problemas psicosociales y de salud relacionados con la inactividad física. 
En relación al modo en el que conocieron el SW, el contacto con amigos o conocidos que ya practicaban el deporte facilitó es el principal medio a través del cual los participantes de nuestra muestra conocieron el deporte y junto con el acceso a las redes sociales. Estos dos elementos se consolidan en nuestra muestra como las principales vías de difusión de este deporte y de interacción con otros practicantes. Además, se refleja la relevancia del apoyo social, ya que la presencia de amigos o conocidos se vincula y puede ser determinante en la iniciación de la actividad física, influyendo de forma directa al proporcionar información sobre la actividad (Serra, Zaragoza y Generelo, 2014).

Acerca de los motivos de inicio y permanencia, los participantes de nuestro estudio consideran los motivos relacionados con la forma física y el disfrute con la actividad como los principales, lo que según otros estudios han sido señalados como predictores de la participación y de la continuidad (Allender, Cowburn and Foster, 2006; Moreno et al., 2005; Torres, Carrasco y Medina, 2010). En la iniciación adicionalmente adquieren relevancia motivos relacionados con la preocupación por el aspecto físico, lo que refleja el enfoque basado en el binomio entre la condición física y la apariencia en nuestra muestra, que son congruentes con los hábitos de vida saludables que promueven (Moreno et al., 2014). Observamos, por lo tanto, un equilibrio entre lo estético y la búsqueda de mejorar la forma física.

En relación a la cohesión, los participantes de este estudio presentan fuertes sentimientos de pertenencia e identificación con el grupo de entrenamiento, satisfacción con el mismo, compromiso con las reglas de funcionamiento establecidas por el grupo, además de sentirse valorados por el resto de los compañeros de entrenamiento. Estas puntuaciones son congruentes con los valores sociales que transmite el SW y que promueven sus representantes, aquellos relacionados con la ayuda mutua y el compañerismo (WSWCF, 2017) en el que personas de distintos orígenes tienen una objetivo común y trabajan en conjunto para conseguir sus objetivos: la práctica de Street Workout y el progreso en este deporte.

En relación a la satisfacción muscular, también acorde con la filosofía que transmiten, se observa una tendencia de interés moderado y de aceptación por el desarrollo de su musculatura y el rechazo generalizado del uso de sustancias como medio para conseguir el desarrollo muscular rápido y extremo, (WSWCF, 2017; FESW, 2017). Los datos no reflejan indicios o riesgos de desarrollar una dismorfia muscular, es decir una preocupación excesiva con el tamaño y forma de sus músculos entre los participantes (Cantón, 2010; González-Martí et al., 2012).

El bienestar psicológico de los participantes de este estudio es alto y sigue la línea de otras investigaciones que afirman que el efecto positivo sobre ésta del entrenamiento en actividades de fuerza aporta vigor, autoestima y confianza, (Warburton, Gledhill y Quinney, 2001). Así, los participantes de este estudio muestran de interés por su progreso y evolución (crecimiento personal), actitudes positivas hacia sí mismos (aceptación), presencia de objetivos y metas (propósito de vida), relaciones calidad y cercanas con otros (relacione positivas), 
así como respeto por la propia individualidad (autonomía). Lo que encaja con las descripciones que sus propios practicantes hacen del deporte como una actividad en la que se promueven valores como la constancia, el esfuerzo y la superación personal. En términos generales, y de acuerdo con Arruza et al. (2008), la práctica deportiva tiene un efecto positivo sobre la percepción de la forma física, así como en la liberación de endorfinas, lo que repercute también en el estado de bienestar; lo que podría incidir en la tendencia observada en este estudio.

Respecto a las diferencias entre grupos, los participantes que compiten presentan una mayor seguridad en sí mismos, se sienten competentes en su interacción con el contexto, poseen propósitos de vida y muestran compromiso con las normas establecidas, en mayor medida que aquellos que no compiten. Estos datos son congruentes con la tendencia perfeccionista de los competidores, concretamente los fisicoculturistas, descritos como más realistas, prácticos y orientados a la acción (Arbinaga y Caracuel, 2007). Características que podrían explicarse por la necesidad de una mayor preparación por parte de los deportistas competidores, frente a aquellos que practican el deporte por motivos relacionados mayoritariamente con la salud. En el caso de nuestros participantes podría ocurrir de manera similar, ya que las competiciones requieren un determinado nivel y, por ende, mejor preparación física, lo que requiere mayor entrenamiento y dedicación.

Por otro lado, la ausencia de una diferencia entre inmigrantes y no-inmigrantes nos hace pensar que este grupo puede considerarse como integrado, ya que de acuerdo con Yañez y Cárdenas (2010), aquellos inmigrantes que logran integrarse en el país de acogida reportan mejores indicadores de bienestar psicológico que aquellos que no lo han logrado. En el caso de nuestra muestra, el nivel general de bienestar es óptimo, independientemente del origen de los participantes, lo cual equipara a ambos grupos.

Estos resultados son congruentes con las características mismas del Street Workout y la filosofía que promueve: un estilo de vida saludable ligado a una condición física óptima que se consigue a través del entrenamiento regular, una alimentación equilibrada y el rechazo activo del consumo de sustancias nocivas para la salud o prohibidas por la WADA (WSWCF; 2017). De igual modo, las características más visibles del SW, como lo son los ejercicios y acrobacias, hacen que sea una actividad atractiva al público, lo que facilitaría la iniciación y repercutiría en su mantenimiento (Torres et al, 2010), explicando así su creciente popularidad.

Las limitaciones que han condicionado el estudio realizado son la falta de equidad entre los grupos de comparación, así como la distribución en función del sexo, que ha impedido que hiciéramos comparaciones en este aspecto y exploráramos los efectos de interacción que pudieran existir. Además, el tipo de muestreo no permite la generalización sobre la población, por lo que líneas futuras de investigación tendrían que tener en cuenta este aspecto, así como las variables fisiológicas que complementen a las variables psicológicas estudiadas. 


\section{CONCLUSIÓN}

Este estudio nos ofrece información sobre las características psicológicas de los practicantes de Street Workout en nuestra muestra contribuyendo a aumentar la escasa bibliografía científica sobre este tipo de modalidades y propone futuras líneas de investigación.

Los practicantes de SW del presente estudio presentan unos niveles óptimos de bienestar psicológico, satisfacción muscular y cohesión, cuyos motivos de iniciación y adición a la práctica deportiva presentan un equilibrio entre la preocupación física, la salud y aspectos motivacionales intrínsecos, como la diversión que produce la práctica en sí. Con base en la capacidad de cualquier otro deporte o ejercicio físico y los resultados expuestos, suponemos que la tendencia de la práctica regular de SW será de influir positivamente en la percepción de la habilidad deportiva y en los aspectos de la condición física que repercuten en el bienestar, así como en la mejora de la calidad de vida y la salud mental y física.

La sociedad puede apostar por estas nuevas modalidades deportivas que están resultando tan atractivas, fundamentalmente para los más jóvenes, ayudando así a la expansión de estilos de vida saludables y a la lucha contra el sedentarismo y sus consecuencias derivadas, entre ellas la obesidad, la diabetes - los problemas cardiovasculares. Asimismo, parece ser una actividad útil y facilitadora de la integración social, empleando además los recursos urbanos ya disponibles, lo que elimina una posible barrera económica para la participación de cualquier persona.

\section{REFERENCIAS BIBLIOGRÁFICAS}

Acero, A., Moreno, G., Moreno, J. y Sánchez, L. (2002). La prevención de las drogodependencias en el tiempo de ocio. Manual de Formación. Madrid: JUMA. ISBN: 84-688-0405-3

Allender, S., Cowburn, G. y Foster, C. (2006). Understanding participation in sport and physical activity among children and adults: a review of qualitative studies. Health Education Research, 21(6), 826-835.

https://doi.org/10.1093/her/cyl063

Andrade, E.M., Arce, C. y Seoane, G. (2002). Adaptación al español del cuestionario "Perfil de los Estados de Ánimo" en una muestra de deportistas. Psicothema, 14, 708-713.

Arbinaga, F. y Cantón, E. (Coordinadores). (2013). Psicología del deporte y la salud: una relación compleja. Madrid: Editorial EOS. ISBN: 978-84-9727506-4.

Arbinaga, F. y Caracuel, J.C. (2007). Dependencia del ejercicio en fisicoculturistas competidores evaluada mediante la Escala de Adicción General Ramón y Cajal. Universitas Psychologica, 6(3), 549-558.

Asociación Cultural y Recreativa Street Workout Zaragoza (2015). Asociación Cultural y Recreativa Street Workout Zaragoza. Recuperado de https://www.heraldo.es 
Arruza, J., Arribas, S., Gil De Montes, L., Irazusta, S., Romero, S. y Cecchini, J.A. (2008). Repercusiones de la duración de la Actividad Físico-deportiva sobre el bienestar psicológico. Revista Internacional de Medicina $y$ Ciencias de la Actividad Física y el Deporte, 8(30), 171-183.

Blacklock, R., Rhodes, R. y Brown, S. (2007). Relationship between regular walking, physical activity and health-related quality of life. Journal of Physical Activity and Health, 4(2), 138-152.

https://doi.org/10.1123/jpah.4.2.138

Cantón, E. (2010). Guía informativa sobre esteroides-anabolizantes. Valencia: Consellería de Sanitat. ISBN: 978-84-482-3916-9.

Caracuel, J. y Arbinaga, F. (2010). Repercusión del ejercicio físico sobre la salud. Apuntes de Psicología, 28, 315-328.

Dean, D., Dean, J. (productores) y Knox, D. (director). (2002). Thug Workout: Fitness From the Streets [DVD]. Estados Unidos: Ventura Distribution.

Díaz, D., Rodríguez-Carvajal, R., Blanco, A., Moreno-Jiménez, B., Gallardo, I., Valle, C. y Van Dierendonck, D. (2006). Adaptación española de las escalas de bienestar psicológico de ryff. Psicothema, 18(3), 572-577.

Djalal, D. (2015). The Socio-economic Value of Sports: Strengthening Social Capital through Football. Journal of Sustainable Development, 8(6).

https://doi.org/10.5539/jsd.v8n6p16

Elling, A. y Claringbould, I. (2005). Mechanisms of inclusion and exclusion in the dutch sports landscape: Who can and wants to belong? Sociology of Sport Journal, 22(4), 498-515.

Federación Española de Street Workout y Calistenia (2017). Los tres pilares de un entrenamiento. Recuperado de: https://www.feswc.com/

García-Ferrando, M. (2006). Veinticinco años de análisis del comportamiento deportivo de la población española (1980-2005). Revista Internacional de Sociología, 64(44), 15-38.

González-Martí, I., Fernández, J., Contreras, O. y Mayville, S. (2012). Validation of a spanish version of the muscle appearance satisfaction scale: Escala de satisfacción muscular. Body Image, 9(4), 517-523.

https://doi.org/10.1016/j.bodyim.2012.05.002

Gutiérrez Sanmartín, M. (2004). El valor del deporte en la formación integral del ser humano. Revista de Educación, 335, 105-126.

Heinemann, K. (2002). Deporte para inmigrantes: instrumento de integración. Apunts. Educación Física y Deportes, 68, 24-35.

Huebner, E. (2004). Research on assessment of life satisfaction of children and adolescents. Social Indicators Research, 66(2), 3-33.

https://doi.org/10.1023/B:SOCl.0000007497.57754.e3

Jiménez, A. (2006). Entrenamiento de Fuerza y Salud: Efectos Positivos Positivos de los Cambios producidos por el Entrenamiento de Fuerza sobre la Salud. PubliCE Standard. Recuperado de: http://g-se.com/

Linares-Manrique, M., Linares-Girela, D., Schmidt-Rio-Valle, J., Mato-Medina, O., Fernández-García, R. y Cruz-Quintana, F. (2016). Relación entre autoconcepto físico, ansiedad e IMC en estudiantes universitarios mexicanos. Revista Internacional de Medicina y Ciencias de la Actividad Física y el Deporte, 16 (62), 497-519.

Maza, G., Balibrea, K., Camino, X., Durán, L., Jiménez, P. y Santos, A. (2011). Deporte, actividad física e inclusión social. Una guía para la intervención 
social a través de las actividades deportivas, Madrid: Consejo Superior de Deportes y Dirección General de Deportes

Monje, J. (2017). Sentido de práctica del Street Workout para los jóvenes escolarizados de la ciudad de Neiva (Tesis de Máster). Universidad de Caldas, Manizales, Colombia.

Moreno, J., Pavón, A., Gutiérrez, M. y Sicilia, A. (2005). Motivaciones de los universitarios hacia la práctica físico-deportiva. Revista Internacional de Medicina y Ciencias de la Actividad Física y el Deporte, 5(19), 154-165.

Moreno-Murcia, J., Sicilia, A., Sáenz-López, P., González-Cute, D., Almagro, B. y Conde, C. (2014). Análisis motivacional comparativo en tres contextos de actividad física. Revista Internacional de Medicina y Ciencias de la Actividad Física y el Deporte, 14(56), 665-685.

Pérez, C. (2016). Análisis sociológico del Street Workout. Trabajo presentado en Sociología y deontología de la Actividad Física y el deporte, Universidad Politécnica de Madrid, Madrid, España.

Pulgar, S. y Fernández-Luna, A. (2019). Práctica de actividad física, consumo de tabaco y alcohol y sus efectos en la salud respiratoria de los jóvenes universitarios. Retos, 35, 130-135.

Radio Television Española. (Productor). (2014) La Aventura del saber. Barbarrio [Reportaje] Disponible en http://www.rtve.es

Ramos, J.A., Sansebastián, J. y Madoz, A. (2001). Desarrollo, validez y seguridad de una escala de adicción general: un estudio preliminar. Actas Españolas de Psiquiatría, 29 (6), 368-373.

Rodríguez, A. y Esnaola, I. (2009). Los trastornos de la conducta alimentaria y el autoconcepto físico. En A. Goñi (Coordinador), El autoconcepto físico: Psicología y educación (pp. 173-192). Madrid: Pirámide.

Rodríguez, J. (2007). Vigorexia: Adicción, obsesión o dismorfia; un intento de aproximación. Salud y Drogas, 7(2), 289-308.

Serra, J., Zaragoza, J. y Generelo, E. (2014). Influencias de "otros significativos" para la práctica de actividad física en adolescentes / Influences from "other influencers" for physical activity practice in teenagers. Revista Internacional de Medicina y Ciencias de la Actividad Física y el Deporte, 14(56), 735-753.

Sicilia, A., Águila, C., Muyor, J., Orta, A. y Moreno, J. (2009). Perfiles motivacionales de los usuarios en centros deportivos municipales. Anales de Psicología, 25(1), 160-168.

Stodolska, M. y Alexandris, K. (2004). The role of recreational sport in the adaptation of first generation immigrants in the United States. Journal of Leisure Research, 36(3), 379-413.

https://doi.org/10.1080/00222216.2004.11950029

Taipe, N., Peris, D. y Cantón, E. (2019). Motivos de práctica deportiva, bienestar psicológico e inmigración en practicantes de Street Workout. Revista de Psicología del Deporte, 28 (2), 135-142.

Taverno, S., Larson, N., Graham, D. y Neumark-Sztainer, D. (2014). Longitudinal changes in physical activity and sedentary behavior from adolescence to adulthood: comparing U.S.-born and foreign-born populations. Journal of Physical Activity \& Health, 11(3), 519-527. http://doi.org/10.1123/jpah.2011-0359 
Torres, G., Carrasco, L. y Medina, J. (2010). Investigación sobre los motivos por los que los estudiantes universitarios practican deporte. El caso de la Universidad de Granada. Motricidad. European Journal of Human Movement, 6(1), 95-105.

UNICEF. (2011). Deporte para el desarrollo integral de la niñez. Disponible en: http://www.unicef.org/costarica/media 21740.htm

Walseth, K. y Fasting, K. (2004). Sport as a means of integrating minority women. Sport in Society, 7(1), 109-129.

https://doi.org/10.1080/1461098042000220218

Wang, D., Wang, Y., Wang, Y., Li, R. y Zhou, C. (2014). Impact of physical exercise on substance use disorders: A meta-analysis. Plos One, 9(10), https://doi.org/10.1371/journal.pone.0110728

Warburton, D., Gledhill, N. y Quinney, A. (2001). The effects of changes in musculoskeletal fitness on health. Canadian Journal of Applied Physiology, 26(2), 161-216.

https://doi.org/10.1139/h01-012

World Street Workout and Calisthenics Federation. (2017). About World Street Workout and Calisthenics Federation. Recuperado de: http://www.wswcf.org/

Yañez, S. y Cárdenas, M. (2010). Estrategias de Aculturación, Indicadores de Salud Mental y Bienestar Psicológico en un grupo de inmigrantes sudamericanos en Chile. Revista Salud y Sociedad, 1(1), 51-70.

https://doi.org/10.22199/S07187475.2010.0001.00006

Zaragoza, J., Serrano, E. y Generelo, E. (2004). La medición de la condición física saludable: aplicación de la batería Eurofit para adultos. Lecturas: Educación Física y Deportes, 68. Recuperado de: http://www.efdeportes.com/

Número de citas totales / Total references: 44 (100\%)

Número de citas propias de la revista / Journal's own references: $5(11,36 \%)$

Rev.int.med.cienc.act.fís.deporte - vol. 20 - número 79 - ISSN: 1577-0354 